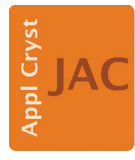

JOURNAL OF

APPLIED

CRYSTALLOGRAPHY

ISSN 1600-5767

Received 6 April 2015

Accepted 12 October 2015

Edited by E. P. Gilbert, ANSTO, Kirrawee DC, Australia

Keywords: block copolymers; crystallization from solution; small-angle neutron scattering; optical microscopy.

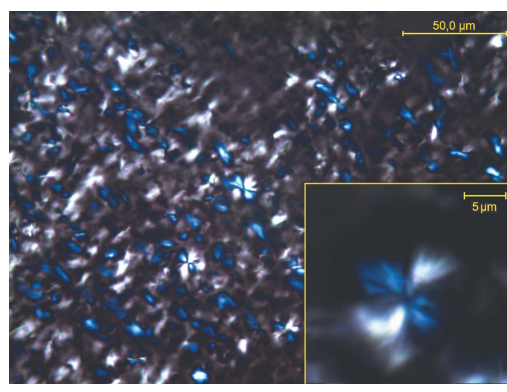

OPEN $\odot$ ACCESS

\section{Morphology of crystalline-amorphous olefin block copolymers in solution characterized by small-angle neutron scattering and microscopy}

\author{
Aurel Radulescu, ${ }^{\text {a* Günter Goerigk, }}{ }^{\mathrm{b}}$ Lewis Fetters $^{\mathrm{c}}$ and Dieter Richter ${ }^{\mathrm{d}}$ \\ aülich Centre for Neutron Science - Outstation at MLZ, Forschungszentrum Jülich GmbH, Lichtenbergstrasse 1, \\ Garching, 85747, Germany, ${ }^{\mathbf{b}}$ Institute of Soft Matter and Functional Materials, Helmholtz Zentrum Berlin für Materialien \\ und Energie, Berlin, 12489, Germany, ' School of Chemical and Biomolecular Engineering, Cornell University, Ithaca, NY \\ 14853-5201, USA, and Jülich Centre for Neutron Science (JCNS-1) and Institute for Complex Systems (ICS-1), \\ Forschungszentrum Jülich $\mathrm{GmbH}$, Jülich, 52425, Germany. *Correspondence e-mail: a.radulescu@fz-juelich.de
}

The single-chain properties and self-assembly behavior in dilute solution of olefin block copolymers obtained by chain-shuttling technology and consisting of alternating crystallizable and amorphous ethylene/1-octene blocks were investigated by pinhole and focusing small-angle neutron scattering techniques, optical microscopy in bright-field and crossed-polarizer modes, and differential scanning calorimetry. The complex hydrocarbon soluble (precipitant free) macro-aggregates formed with decreasing temperature are characterized by spherulitic textures. The spherulites yield, on one hand, a morphology that depends on the chain structure properties and, on the other hand, multiple structural levels with a hierarchical organization that ranges from $10 \AA$ up to tens of micrometres. This morphology displays peculiarities dictated by the polydisperse character of these materials.

\section{Introduction}

The macroscopic behavior of crystalline-amorphous polymers depends on the constitutive microstructures, which consist of molecules arranged as unit cells, lamellar crystals, fibrils, boards or spherulites (Akpalu, 2010). These structures can span a wide length scale of several nanometres to hundreds of micrometres. The control and optimization of polymer properties requires the knowledge and understanding of the microstructural properties under various conditions. Recently (Radulescu et al., 2004, 2006, 2011; Radulescu et al., 2008), we have studied the assembly in solution of a long series of semicrystalline ethylene-1-butene copolymers (PEB- $n$ ) with graded ethylene content (where $n$ is the number of ethyl branches per 100 backbone $\mathrm{C}$ atoms) by combining different small-angle neutron scattering techniques and microscopy. Different architectures of these copolymers have been investigated, i.e. PEB- $n$ block copolymers with $n$ ranging between 7.5 (higher crystallinity) and 11 (lower crystallinity), as well as multi-block copolymers built up by PEB- $n$ blocks showing a graded degree of crystallinity. These materials consist basically of microcrystalline ethylene units copolymerized with amorphous butene segments. They exhibit potential as flow improvers for middle distillate refinery fuels as previous structural studies on these wax/PEB- $n$ solutions have concluded (Radulescu et al., 2004; Radulescu, Fetters \& Richter, 2012). Other crystalline-amorphous polymer architectures may also show in solution properties of interest for different applications, particularly as wax crystal modifiers. Recent developments from the DOW Chemical Company 
have led to the synthesis of novel olefinic block copolymers (OBCs) by using chain shuttling technology (Arriola et al., 2006). These are ethylene-octene OBCs and jointly consist of crystallizable blocks (hard) with very low octene co-monomer content and high melting temperature alternating with amorphous blocks (soft) with relatively high 1-octene content. These materials are similar in having a statistical multi-block architecture with distributions both in block length and in the number of blocks per chain (Shan \& Hazlitt, 2007). Thus, by varying the hard block content, a broad range of elastomeric and thermal properties emerge. Combined with the ability to form complex nanoscale morphologies, these OBCs enable a wide range of applications to be accessed (Wang et al., 2009). Owing to the block length polydispersity the OBCs exhibit much larger domain spacing than the traditional block copolymers of similar molecular weight. Unlike the statistical copolymers that form fringed micellar crystals, the OBCs can form space-filling spherulites even for low-crystalline-content material (Wang et al., 2007). The relationship between the structure and morphology and the crystallization behavior in OBCs, which governs the properties of these materials, remains incompletely explored. Particularly, the assembly properties of these materials in solution have not yet been studied.

Our general aim was to investigate the structural properties of the aggregates yielded in dodecane solution by two lowcrystallinity OBCs during the cooling of solutions between the polymer single-coil regime, at high temperatures, and $293 \mathrm{~K}$. The complex morphologies of the OBC aggregates, displaying multiple structural levels spanning a wide length scale, between $10 \AA$ and several micrometres, have been characterized by combining a multitude of techniques: pinhole smallangle neutron scattering (SANS), focusing small-angle neutron scattering (f-SANS), differential scanning calorimetry (DSC) and optical microscopy with crossed polarizers.
(293-378 K). The $1 \%$ polymer solutions at $293 \mathrm{~K}$ were additionally investigated by f-SANS.

The SANS measurements were performed at the KWS-2 classical pinhole SANS (Radulescu, Pipich \& Ioffe, 2012; Radulescu, Pipich et al., 2012) and KWS-3 mirror-focusing f-SANS (Goerigk \& Varga, 2011) diffractometers of the Heinz Maier-Leibnitz Centre (MLZ) at the FRM II reactor in Garching, München (Gläser \& Petry, 2000). In pinhole geometry, using three detection distances $(2,8$ and $20 \mathrm{~m})$ and a neutron wavelength $\lambda=7 \AA(\Delta \lambda / \lambda=20 \%)$ at KWS-2, a wavevector transfer $Q$ range between $1.5 \times 10^{-3}$ and $0.2 \AA^{-1}$ was covered. At KWS-3, two sample-to-detector distances ( $\mathrm{SD}=1.5$ and $10 \mathrm{~m}$ ) and the neutron wavelength $\lambda=12 \AA$ $(\Delta \lambda / \lambda=20 \%)$ were used, which allowed us to perform measurements within a $Q$ range between $1.2 \times 10^{-4}$ and $0.02 \AA^{-1}$. The special design of the f-SANS instrument, based on the one-to-one mirroring of a small entrance aperture on a high-resolution $(0.4 \mathrm{~mm})$ position-sensitive detector by using a double focusing toroidal mirror, enables one to reach such low $Q$ values with still sufficient intensity. In both cases, the data were corrected for detector sensitivity, instrumental noise and scattering from the empty cell, then radially averaged and calibrated in absolute units using a Plexiglas standard sample (Radulescu, Pipich et al., 2012). The results were subsequently corrected for scattering from solvent.

Thus, the combination of the two techniques allowed us to investigate structural sizes from $10 \AA$ to $1 \mu \mathrm{m}$. The polymer single-coil characteristics and self-assembly behavior were determined from the investigation of the scattered intensity by SANS at different temperatures within the range from 413 to $293 \mathrm{~K}$, decreasing the temperature in steps of $20 \mathrm{~K}$.

The polymer aggregates in the $\varphi=1 \%$ solution were finally examined using optical microscopy. Solutions dropped on glass lamellae were observed using the bright-field and crossed-polarizer modes with a Leica DM6000M light microscope with polarization options.

Specimens weighing $4 \mathrm{mg}$ were cut from the original polymer pellets for a supplementary thermal analysis on a PerkinElmer Series 7 differential scanning calorimeter. Scans

\section{Materials and methods}

Two INFUSE OBCs, 9000 and 9007, were obtained as pellets from the DOW Chemical Company. The overall density of the materials is 0.877 and $0.866 \mathrm{~g} \mathrm{~cm}^{-3}$, respectively, as provided by the manufacturer. These OBCs are ethylene-octene multiblock copolymers (Dias et al., 2008; Kamdar et al., 2009) and are shown schematically in Fig. 1. Polymer solutions were prepared in deuterated dodecane (d-26) at $413 \mathrm{~K}$ for polymer volume fractions between $\varphi=0.1$ and $1 \%$. In order to determine the single-coil properties and the self-assembly behavior of the OBCs, the polymer solutions were investigated by SANS over a wide temperature range, between the single-coil regime $(>378 \mathrm{~K})$ and the aggregation regime

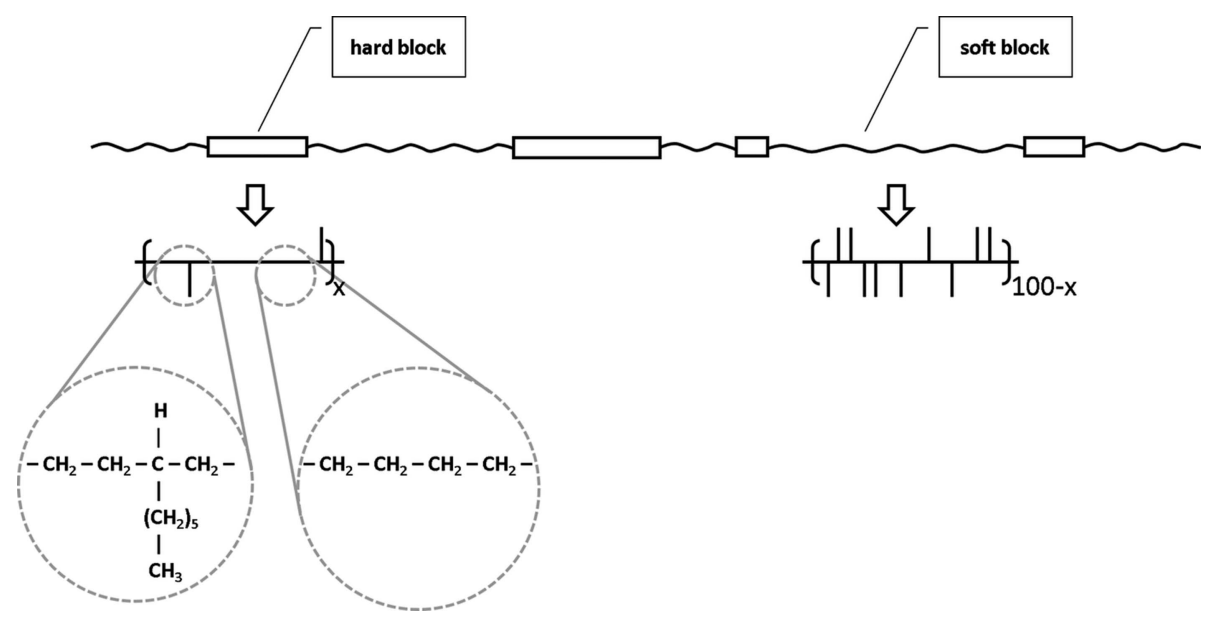

Figure 1

Schematic representation of the OBCs. 


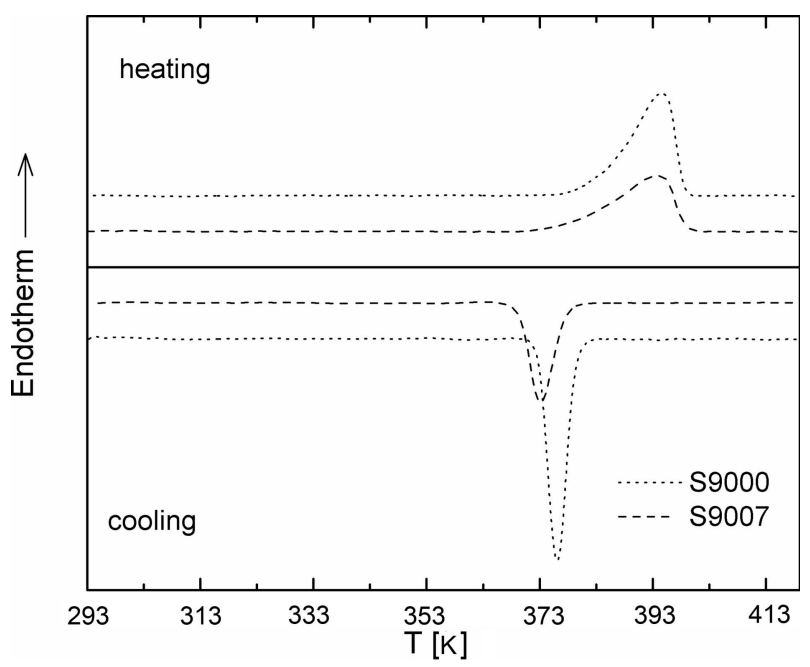

Figure 2

Thermal behavior of OBCs: the melting (top) and crystallization (bottom) processes at a rate of $5 \mathrm{~K} \mathrm{~min}^{-1}$.

were taken under a nitrogen atmosphere between 10 and $423 \mathrm{~K}$ with a heating/cooling rate of $5 \mathrm{~K} \mathrm{~min}^{-1}$.

Additional insight about the morphology and substructure of polymer assemblies was obtained by the examination of the wax decorated polymer aggregates under the microscope, a method used before for the indirect visualization of PEB-7.5 polymeric needles (Radulescu et al., 2006). Polymer-wax mixed assemblies formed upon cooling from $413 \mathrm{~K}$ in common dodecane solution of OBCs with 1 and $4 \%$ hexatriacontane wax $\left(\mathrm{C}_{36}\right)$. These were complementarily examined at $293 \mathrm{~K}$, below the wax precipitation point (Radulescu et al., 2008), with the Leica DM6000M light microscope in bright-field mode.

\section{Results and discussion}

\subsection{Thermal behavior}

The DSC curves of the melting and the subsequent crystallization of the two OBCs are shown in Fig. 2. The values of
Table 1

Characteristics and physical properties of OBCs.

\begin{tabular}{|c|c|c|c|c|c|c|}
\hline OBC & $\begin{array}{l}\text { Density } \dagger \\
\left(\mathrm{g} \mathrm{cm}^{-3}\right)\end{array}$ & $T_{\mathrm{m}}(\mathrm{K})$ & $T_{\mathrm{c}}(\mathrm{K})$ & $\begin{array}{l}X_{\mathrm{c}, \Delta H} \text { \% } \\
(\mathrm{wt} \%)\end{array}$ & $\begin{array}{l}M_{\mathrm{W}} \S \\
\left(\mathrm{kg} \mathrm{mol}^{-1}\right)\end{array}$ & $R_{\mathrm{g}} \S(\AA)$ \\
\hline 000 & 0.877 & 3946 & 376.2 & 12 & 159 & 195 \\
\hline 9007 & 0.866 & 393.8 & 373.2 & 8 & 117 & 196 \\
\hline
\end{tabular}

$\dagger$ Density values taken from the manufacturer data sheets. $\$$ Crystallinity determined by DSC. $\$$ Chain characteristics determined by SANS.

melting temperature $\left(T_{\mathrm{m}}\right)$ and crystallization temperature $\left(T_{\mathrm{c}}\right)$ are summarized in Table 1. The high melting temperature (around $393 \mathrm{~K}$ for both OBCs) is typical for the OBCs in which the long ethylene sequences of the hard blocks crystallize as large, chain-folded lamellae (Wang et al., 2009). Although the position of the melting peak is similar for the two OBCs, the heat of melting taken as the area of the peaks differs sizably. First insights about the crystallinity from heat of melting $X_{\mathrm{c}, \Delta H}$ of the two materials (total hard and soft blocks) were obtained from the direct interpretation of the crystallization peaks following a procedure described elsewhere (Wang et al., 2009). The 9000 OBC presents a higher crystallinity than the $9007 \mathrm{OBC}$, which leads to the conclusion that the global co-monomer content (1-octene) in the 9007 OBC is higher than in the 9000 OBC. This observation is strengthened by the crystallization behavior of the two OBCs, which shows sizable differences both in the position and in the area of the crystallization peaks.

\subsection{Optical microscopy observations}

Fig. 3 shows images of typical aggregates formed by the two OBCs $(\varphi=1 \%)$. These were collected at $293 \mathrm{~K}$ using the bright-field observation mode. Although in both cases large aggregates with sizes of about $10 \mu \mathrm{m}$ are observed, a detailed inspection of the micrographs reveals that the morphologies yielded by the two OBCs in solution differ. In the case of the 9000 OBC, aggregates resembling bundles or sheaves of elongated objects are clearly revealed. The 9007 OBC gives rise to spherical aggregates that show a dense nucleus.

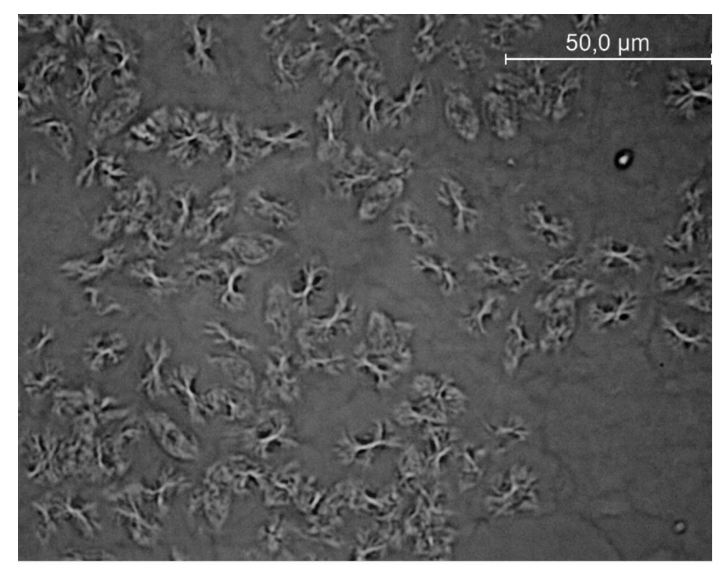

(a)

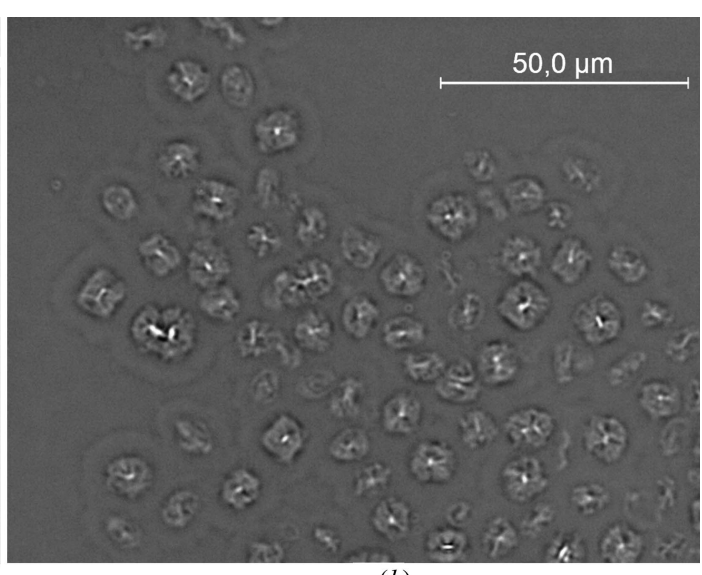

(b)

Figure 3

Micrographs of the assemblies of $9000(a)$ and 9007 (b) OBCs in dodecane observed at $293 \mathrm{~K}$ using bright-field microscopy. 


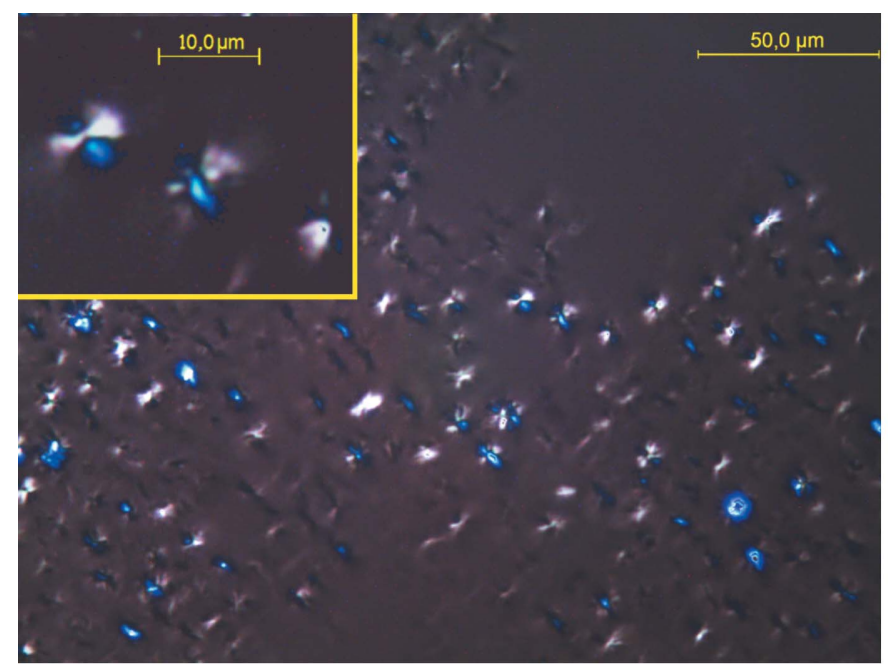

(a)

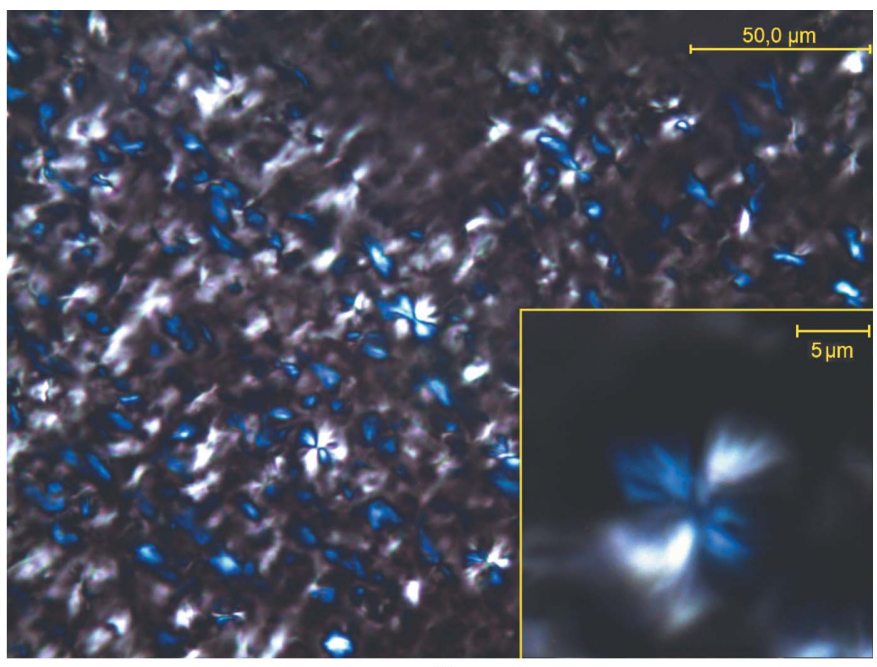

(b)

Figure 4

Crossed-polarized micrographs of the assemblies of $9000(a)$ and 9007 (b) OBCs in dodecane at $293 \mathrm{~K}$.

Observed between crossed polarizers (Fig. 4), both types of macro-aggregates exhibit dark and bright patterns due to birefringence (the 'Maltese cross' feature). This shows that both OBCs can yield in solution crystalline-amorphous spherulites. Typically, a polymer spherulite represents a spheriform cluster of fibrillaror lamellar-like crystallites alternating with amorphous domains which start growing from a nucleation site and splay out laterally (Magil, 2001; Gránásy et al., 2005). Both types of polymer assembly show a hierarchical structural organization.

For the case of the 9000 OBC such a feature is evident by examining the micrographs under higher magnification (Fig. 5a). The sheaf-like morphology exhibits in this case fibrillar-like structural sub-units: a central trunk with a thickness of about $1 \mu \mathrm{m}$, possibly consisting of a bundle of fibrils, which shows at both ends submicrometric scattered branches. For the more homogeneous aggregates formed by the 9007 $\mathrm{OBC}$ no clear features were revealed by higher magnifications and the substructure may be only presumed on the basis of the structural peculiarities typically exhibited by the crystallineamorphous spherulites.

An attempt to observe the substructures of the spherulitic morphology by transmission electron microscopy (TEM) was also made. No obvious morphology or structure was observed by TEM. It seems that either the macro-aggregates observed by optical microscopy in dodecane solutions were easily destroyed by the procedure of the sample preparation for TEM observation or the substructure in the spherulitic

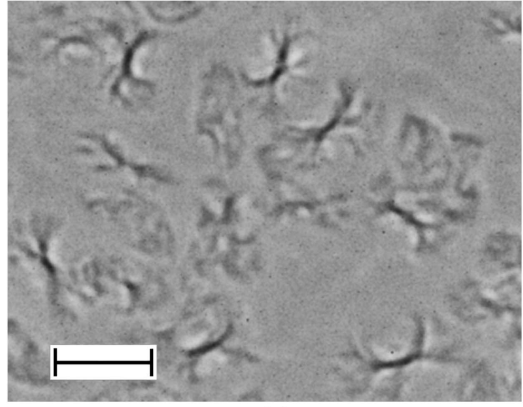

(a)

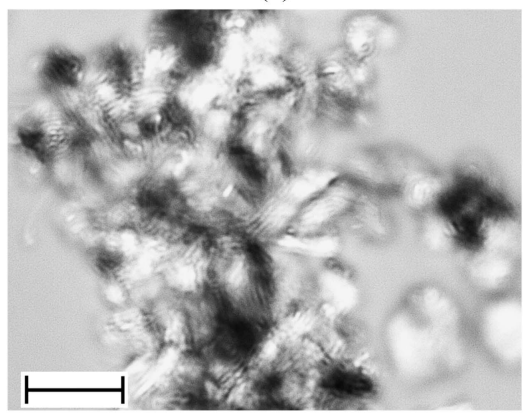

(c)

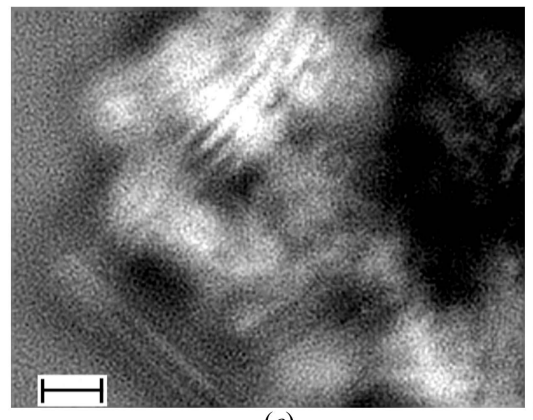

(e)

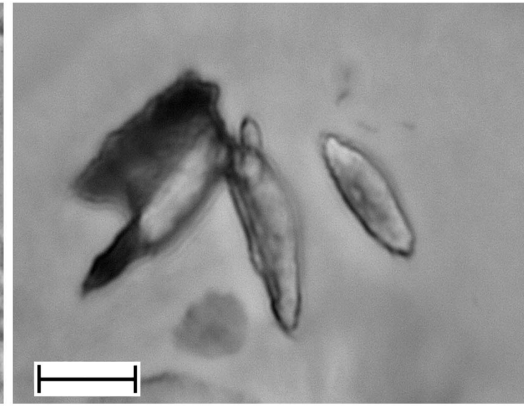

(b)

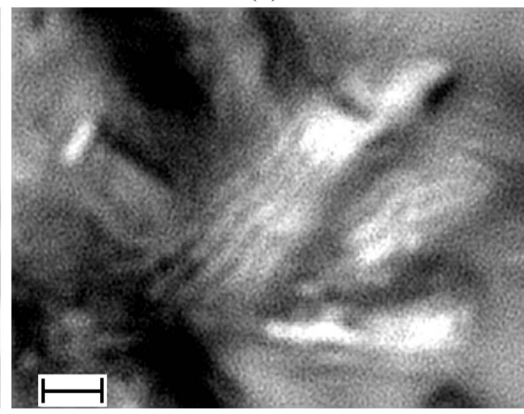

$(d)$

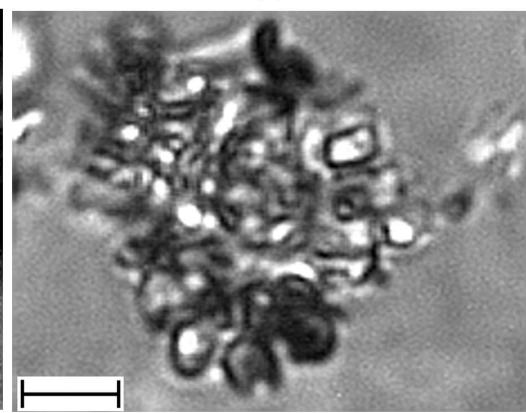

$(f)$
Figure 5

Micrographs of the polymer assemblies and polymer-wax common aggregates in deodecane observed at $293 \mathrm{~K}$ using bright-field microscopy for the $9000 \mathrm{OBC}(a)$, the $9000 \mathrm{OBC}$ and $1 \% \mathrm{C}_{36}$ wax $(b)$, the $9000 \mathrm{OBC}$ and $4 \% \mathrm{C}_{36}$ wax $(c)-(e)$, and the $9007 \mathrm{OBC}$ and $1 \% \mathrm{C}_{36}$ wax $(f)$; scale bar: $10 \mu \mathrm{m}(a)-(c),(f), 2 \mu \mathrm{m}(d),(e)$. 
morphologies cannot be recognized by TEM because they are thicker and visualized through a projection of too many structural elements which averages out all structures in the images.

The substructure of the OBCs' spherulites was highlighted indirectly by decorating the polymeric aggregates with wax crystals formed at lower temperature than the polymer crystallization temperature.

When the $9000 \mathrm{OBC}$ is mixed in common solution with a small wax volume fraction of $\mathrm{C}_{36}$ hexatriacontane $\left(\varphi_{\text {wax }}=1 \%\right)$, the resulting system has a wax crystallization point much lower than the sole OBC's crystallization temperature. Here, small spindle-like wax structures were formed (Fig. $5 b$ ). This crystallization habit differs markedly from the typical 'house-ofcards' wax morphologies several hundreds of micrometres in size (Radulescu et al., 2008) and seems to be a consequence of the wax nucleation and controlled growth onto the polymer sheaf-like overall morphology (Fig. $5 a$ ). In the case of higher wax contents $\left(\varphi_{\text {wax }}=4 \%\right)$, i.e. higher wax crystallization point, larger agglomerates are formed (Fig. 5c). Higher magnification (Fig. $5 d$ and $5 e$ ) reveals that the agglomerates consist of aligned arrangements of parallel needle-like aggregates. The needles are about $10 \mu \mathrm{m}$ long and, taking into account possible off-focusing effects that are inherent at this magnification power, have a thickness of $0.5 \mu \mathrm{m}$ or smaller. The correlation distance between needles seems to be also smaller than $0.5 \mu \mathrm{m}$. Finally, macro-aggregates consisting of agglomerations of smaller platelet-like structures can be observed in the common solution of $9007 \mathrm{OBC}$ and $\mathrm{C}_{36}$ wax for $\varphi_{\text {wax }}=1 \%$ (Fig. 5f). The formation of such a morphology is again a consequence of the crystallization of wax molecules templated by initial polymer aggregates, which in this case appear to be lamellar spherulites.

\subsection{Small-angle neutron scattering}

Figs. $6(a)$ and $6(b)$ display the scattering profiles from the $1 \%$ polymer solutions measured at different temperatures by SANS. The power law exponents $\left(Q^{-p}\right)$ specific for different structures formed by the copolymers are given. Hence, swollen single coils $(p=5 / 3)$, one-dimensional $(p=1)$ and platelet-like $(p=$ 2) morphologies, or smooth interfaces $(p=4)$ can be identified and characterized (Radulescu et al., 2011). The model curves of the single-coil polymer morphologies described later are also depicted.

Within the temperature range from 413 to $283 \mathrm{~K}$ all polymers are dissolved as single coils, as shown by the single-chain form factor features identified in the scattering profiles. The plateau towards low $Q$ yields information about the volume fraction and molecular weight. The bending down of the intensity is characteristic of the Guinier regime, from which information about the radius of gyration of the polymer coil is gleaned. The $Q^{-5 / 3}$ power law regime towards high $Q$ is indicative of excluded volume interactions between the chain segments.

Characterization of the single-chain properties was possible from the analysis of the data measured for different polymer concentrations in terms of the Zimm approximation:

$$
\left[\frac{\mathrm{d} \Sigma}{\mathrm{d} \Omega}(Q) K\right]^{-1}=\frac{1}{V_{\mathrm{W}} \varphi}+2 A_{2}+L_{\mathrm{sl}} Q^{2},
$$

where $K=N_{\mathrm{A}} / \Delta \rho^{2}$ is the inverse contrast factor $\left(N_{\mathrm{A}}\right.$ being the Avagadro constant and $\Delta \rho^{2}$ being the contrast factor between the polymer and the solvent), $V_{\mathrm{W}}$ is the molar volume, $A_{2}$ is the second virial coefficient and $L_{\mathrm{sl}}=R_{\mathrm{g}}^{2} /\left(3 \varphi V_{\mathrm{W}}\right)$, with $\varphi$ and $R_{\mathrm{g}}$ the polymer volume fraction and radius of gyration, respectively.

This analysis (Fig. 7) results in a radius of gyration $R_{\mathrm{g}}$ of 195 (14) $\AA$ for the 9000 OBC and 196 (18) $\AA$ for the 9007 OBC. The analysis of the 'forward scattering' $\mathrm{d} \Sigma / \mathrm{d} \Omega(0)$ as a function of polymer volume fraction resulted in the molar volume $V_{\mathrm{W}}$ which delivered the molecular weights $\left(M_{\mathrm{W}}\right)$ of $158.9 \pm 21.8 \mathrm{~kg} \mathrm{~mol}^{-1}$ and $116.6 \pm 20.4 \mathrm{~kg} \mathrm{~mol}^{-1}$ for the two OBCs. Typically, these INFUSE materials contain chains of two to ten alternating blocks, while the hard block weight average molecular weights range from 7 to $28 \mathrm{~kg} \mathrm{~mol}^{-1}$ (Wang et al., 2009; Kamdar et al., 2009). An overall fit of the singlechain scattering patterns with the Beaucage form factor (Beaucage, 1996) is possible:

$$
\begin{aligned}
\frac{\mathrm{d} \Sigma}{\mathrm{d} \Omega}(Q)= & \sum_{i=1}^{m}\left(G_{i} \exp \left(\frac{-Q^{2} R_{\mathrm{g} i}^{2}}{3}\right)\right. \\
& \left.+B_{i} \exp \left(\frac{-Q^{2} R_{\mathrm{g}(i+1)}^{2}}{3}\right)\left\{\frac{\left[\operatorname{erf}\left(Q R_{\mathrm{g} i} / 6^{1 / 2}\right)\right]^{3}}{Q}\right\}^{p_{i}}\right)
\end{aligned}
$$

For a single structural level $(m=1)$ the use of the parameters from the Zimm analysis (the 'forward scattering' $G$ and $R_{\mathrm{g}}$ ) and an exponent $p=5 / 3$ indicative of swollen single chains was 
successful, as one can see in Fig. 6. Following the assumption of a 'polymeric constraint' (Beaucage, 1996; Hammouda, 2010) the parameter $B$ in equation (2) was expressed in terms of $G, R_{\mathrm{g}}$ and $p$, hence reducing the number of free parameters.

A decrease in the temperature resulted in the formation and evolution of polymer aggregates that led to an increase of the scattering level towards low $Q$ compared to the singlechain scattering feature. For both OBCs the first aggregation indications were observed in the scattering data at $378 \mathrm{~K}$. The scattered intensity increases monotonically up to $293 \mathrm{~K}$ (not shown in Fig. 6), indicating that the aggregates grow in size and number.

The scattering patterns are characterized by power law regimes with characteristic exponents for the morphologies formed.

The self-assembly behavior of the two OBCs at the length scale between 10 and $1000 \AA$ (SANS range) is very similar. The scattering profiles from both materials in the aggregation regime (Figs. 6) display the same peculiarities: a seemingly $Q^{-2}$ power law behavior of the scattered intensity and a peaklike feature in the low- $Q$ range of the SANS domain.

The $Q^{-2}$ power law indicates the formation of two-dimensional lamellar-like aggregates. The peak-like feature may denote either a large interlamellar domain spacing or the presence of a higher-order structure. Higher-order scattering maxima are not usually seen because of the washing out of the fine structure resulting from the distribution of spherulite size or disorder. From the analysis of the microscopy images and the TEM attempts no peculiarities of higher-order structure were observed. On the other hand, it is known that, in block copolymers, increasing polydispersity is expected to lead to substantially increased domain periodicity compared with near-monodisperse block copolymers having similar chemical composition. The interlamellar domain spacing can be calculated according to $D^{*}=2 \pi / Q^{*}$, where $Q^{*}$ represents the peak position. These characteristics are typical for a morphology

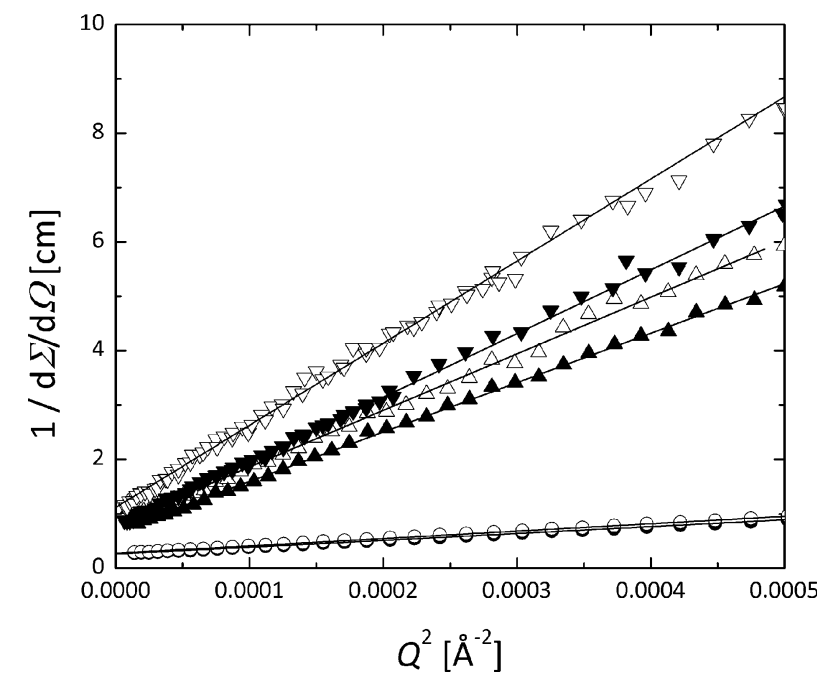

Figure 7

Zimm plot of the scattering from single chains of 9000 (filled symbols) and 9007 (open symbols) OBCs in d-26 at $403 \mathrm{~K}$, for different polymer concentrations ( $\varphi=1,0.2$ and $0.1 \%$ from bottom to top). made of crystalline lamellae alternating with amorphous interlamellar regions similar to that reported in the case of crystallization of OBCs from the melt (Wang et al., 2007, 2009). With decreasing temperature a weak shift of the peak position towards lower $Q$ values is observed, which indicates an increase of the interlamellar spacing. The estimated interlamellar distances at $293 \mathrm{~K}, D^{*} \simeq 1700 \AA$ and $D^{*} \simeq 2300 \AA$ for the 9000 and 9007 OBCs, respectively, are very large, untypical for traditional block copolymers of similar molecular weights (Green, 2001).

On the other hand, the bending down of the scattering patterns at $293 \mathrm{~K}$ observable at $Q \simeq 0.05 \AA^{-1}$ and the weak hump-like structural feature noticeable towards high $Q$ resemble the form factor details of a much thinner density profile perpendicular to the lamellar surface, which seems to correspond to the thickness of the crystalline lamellar core. From the presentation of the scattering data in a Kratky-type plot (Fig. 8) the thickness $d$ of the crystalline lamellae can be extracted in a direct fashion through $Q_{\min }=2 \pi / d$.

Using this crude approach a lamellar thickness of about $100-110 \AA$ is roughly estimated for both OBCs. This very low value compared to $D^{*}$ indicates that either the amorphous interlamellar regions are very thick or crystalline-amorphous morphologies well separated from one another are kept together in a correlated arrangement (stack) by a special mechanism. The combined SANS and microscopy findings lead to the conclusion that the lamellar stacks may consist of correlated thin elongated crystalline lamellae surrounded on both faces by amorphous layers (core-brush morphology), which are well separated from each other by regions characterized by very low polymer content.

Such morphology would offer in a common solution with wax molecules a nucleation platform for wax crystal formation at low temperature, on one hand, and a control mechanism of a limited wax crystal growth through the polymer brush within the amorphous region, on the other hand. The mechanism would be similar to that observed in the case of wax crystal-

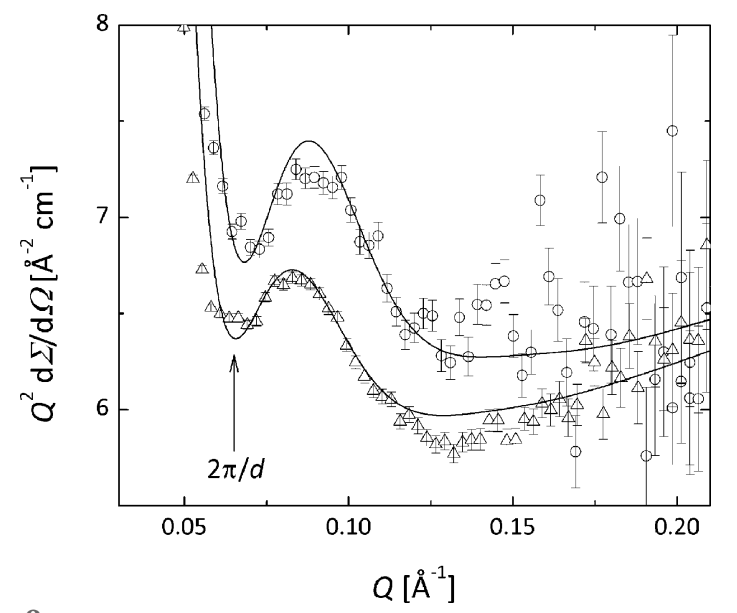

Figure 8

Kratky plot of the scattering in the high- $Q$ region from the assemblies of 9000 (open triangles) and 9007 (open circles) OBCs in d-26 at $293 \mathrm{~K}(\varphi=$ $1 \%$ ); the curves represent the model fit of the polymer morphologies (see text). 


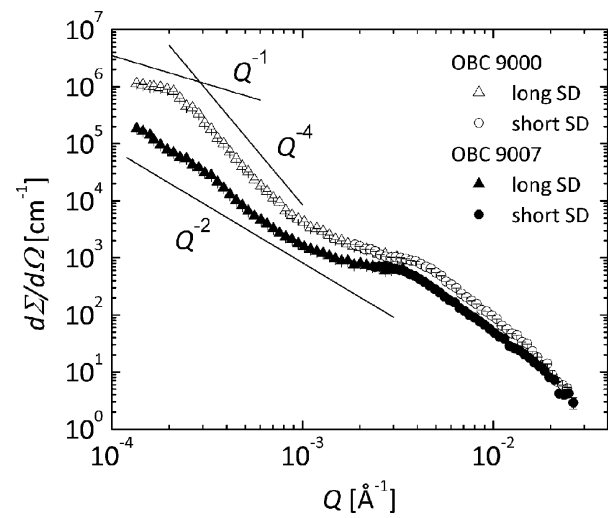

Figure 9

Small-angle neutron scattering cross sections from solutions of 9000 and 9007 OBCs in d-26 $(\varphi=1 \%)$ measured at $293 \mathrm{~K}$ using f-SANS. The solid lines indicate the power-law behavior in the low $Q$ range. Data collected at two sample-to-detector (SD) distances are shown.

lization on polyethylene-poly(ethylene-propylene) PE-PEP (Richter et al., 1996) or PEB-7.5 (Radulescu et al., 2004) crystalline-amorphous two-dimensional morphologies. Wax crystal morphologies such as those observed in Figs. 5(b)-5(f) can be explained in this case by the templation and control of wax crystal growth via peculiarities of the primordial polymer aggregates.

Fig. 9 shows the f-SANS scattering profiles from the two OBCs at $293 \mathrm{~K}$. Although the peak-like features are smoother owing to the resolution, the data collected at the short detection distance are similar to the low- $Q$ data measured by conventional pinhole SANS. The very low $Q$ patterns are significantly different for the two polymers. The $Q^{-2}$ power law in the case of the $9007 \mathrm{OBC}$ extends down to the lowest $Q$ value, displaying a kind of shoulder, which suggests a bimodal size distribution. The observed shoulder at $Q \simeq 0.00035 \AA^{-1}$ could be indicative of the thickness of a lamellar stack. The extended $Q^{-2}$ behavior indicates that the lamellar morphologies show lateral extensions up to $5000 \mathrm{~nm}$, thereby covering

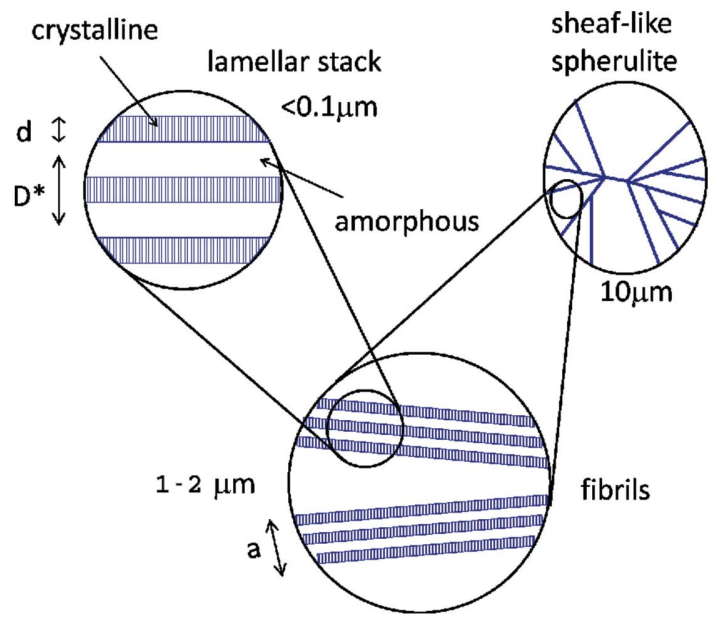

(a) a length scale comparable to the radius of the spherulites observed by optical microscopy (Fig. $3 b$ ).

In contrast, the 9000 OBC scattering pattern exhibits towards low $Q$ a steep power law $(p=4)$ and terminates towards the lowest $Q$ in a Guinier-like regime. Here, the commencement of an apparent $Q^{-1}$ power law and thus the formation of the large-scale fibrillar-like structures revealed by the optical microscopy observations may be presumed. An analysis of the low- $Q$ data in terms of the one-dimensional Guinier approximation (Schwahn et al., 2002) revealed a rod (fibril) thickness of about $a=1.5 \mu \mathrm{m}$, which is quite consistent with the optical microscopy observations.

On the basis of the qualitative information delivered by DSC, microscopy and wide- $Q$-range SANS a hierarchical morphology of the macro-aggregates formed by the two OBCs in solution is schematically proposed in Fig. 10. The aggregates show multiple structural levels and a hierarchical organization on a length scale from $10 \AA$ up to tens of micrometres. In the case of the OBC with higher crystallinity (the 9000 OBC) a crystalline-amorphous primary morphology consisting of thin lamellar slabs (core-brush morphology) arranged in stacks and characterized by a large interlamellar spacing was formed. The correlated lamellae grow mostly longitudinally, exhibiting at a larger length scale a board-like or one-dimensional overall aspect (Schmidt-Rohr, 2007). These are the nucleation platforms for crystallization of wax molecules from common polymer-wax solutions, which template the correlated arrangements of parallel needle-like structures observed in Figs. 5(d) and 5(e). A lamellar crystal is typically formed as a result of the tendency of crystallizable segments to attach progressively to a preferred growth plane. Upon cooling of the OBCs, the crystallization of the hard blocks forces the segregation of the non-crystallizable blocks into the interlamellar regions (Wang et al., 2009). A good separation of the hard block lamellar crystals from the interlamellar soft blocks appears to be present even in the copolymers with very low hard block content (Wang et al., 2007).

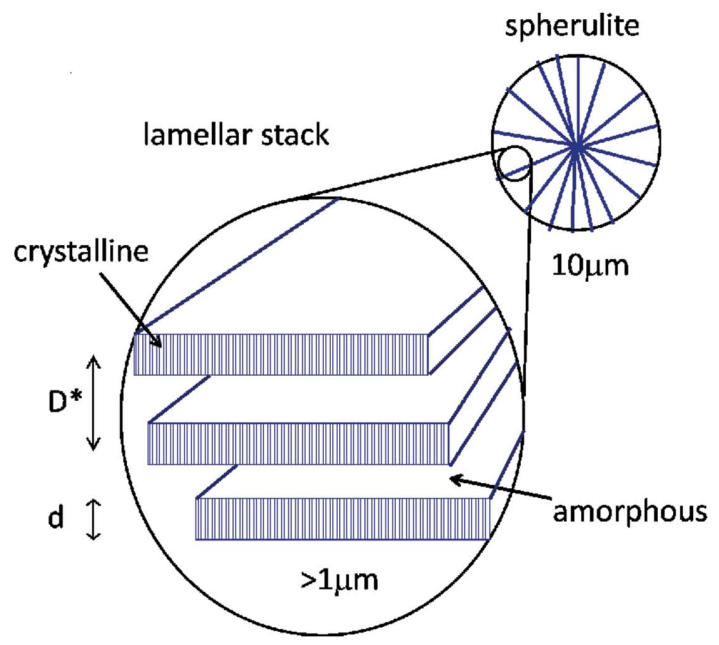

(b)

Figure 10

The hierarchical organization of the main structural levels displayed by the macro-aggregates formed by the $9000(a)$ and $9007(b)$ OBCs in solution, as emerged following the interpretation of the scattering data and DSC and microscopy observations. 
The combination of SANS and microscopy offered a unique method for a complete understanding of the complex morphology in the case of the 9000 OBC: the thin crystalline lamellae surrounded on both sides by amorphous layers are separated from each other by a third layer of a very low material content. The stacked elongated lamellae of the 9000 $\mathrm{OBC}$ give rise to a secondary fibril-like morphology with a lateral size within the micrometre scale. Wax crystals formed at high temperature (high wax content) decorate this morphology and emphasize it under the optical microscope (Fig. $5 d$ and 5e). The fibrils associate in bundles and branch, leading to the formation of the tertiary morphology represented by the sheaf-like spherulites observed in Fig. 5(a). Crystallization of wax molecules at low temperature (low wax content) on the skeleton of such polymeric structures gives rise to the formation of spindle-like crystals (Fig. 5b).

In the case of the $9007 \mathrm{OBC}$ (the material with lower crystallinity) correlated lamellae with a very large lateral extension apparently grow from nucleation sites and splay out in fan-like spherulitic morphologies, dominated by large amorphous regions. This is the typical case when a spherulitic morphology results from the pressure of non-crystallized segments emerging from the lamellar surfaces, which impose the divergent arrangement of the lamellae. This can explain the more regular and homogeneous aspect of the macroaggregates observed by bright-field microscopy (Fig. 3b), which dictates also the peculiarities of the wax crystallization from a common polymer-wax solution (Fig. $5 f$ ).

Although both OBCs show similar structural features at the sub-micrometre length scale, their structural features at the micrometre length scale and the overall aspects (Fig. 10) are very different. This behavior appears to be a consequence of the chain characteristics of the two polymers (Fig. 1). The material with higher 1-octene content (longer sequences with side groups), 9007 OBC, yields the more regular and homogeneous morphologies, with preponderant amorphous behavior. The large platelets schematically depicted in Fig. 10(b) should be seen as isolated lamellae surrounded on both sides by broad amorphous phases. It is worth noticing (Wang et al., 2007) that the lamellae have a short and discontinuous character. Conversely, the formation of irregular bundle- and sheaf-like spherulitic morphologies is favored by the 9000 OBC sample (longer and/or frequent crystallizable sections in Fig. 1). Typically, the sheaf-like type of spherulite is thought to be the result of homogeneous nucleation when a single needle is formed and subsequently branches. The $9000 \mathrm{OBC}$ morphology seems to arise as a consequence of homogeneous nucleation and growth of fibril-like structures accompanied or followed by frequent co-crystallization and cross-linking events occurring between these structures.

A quantitative characterization of the lamellar stacks was done by fitting the SANS data $\left(Q>0.001 \AA^{-1}\right)$ in terms of the structural model of correlated crystalline lamellae surrounded on both sides by amorphous layers coexisting with single chains in solution. This approach was successfully used for the characterization of the lamellar assemblies of PE-PEP crystalline-amorphous diblock copolymers (Richter et al., 1996) and the lamellar crystals of PE (Wang, 2004) in solution. The model combines the form factor of individual crystallineamorphous lamellar slabs (core-brush morphology) with the paracrystalline structure factor describing the stacking effects [equation (3)] over distances larger than the thickness of the lamellar slabs:

$$
S(Q)=\frac{\sinh \left(Q^{2} \sigma_{D *}^{2} / 4\right)}{\cosh \left(Q^{2} \sigma_{D *}^{2} / 4\right)-\cos \left(Q D^{*}\right)},
$$

with $\sigma_{D^{*}}$ the smearing parameter of $D^{*}$. As long as the lateral extension of lamellae and lamellar thickness are well separated in length scale, which is the case for the examination of the SANS data, the scattering cross section may be expressed as

$$
\frac{\mathrm{d} \Sigma}{\mathrm{d} \Omega}(Q)=\frac{\varphi_{\mathrm{sl}}}{Q^{2}} P(Q) S(Q)+\left(\frac{\mathrm{d} \Sigma}{\mathrm{d} \Omega}\right)_{\mathrm{exc}},
$$

where $\varphi_{\mathrm{sl}}$ is the volume fraction of lamellar slabs, $P(Q)$ the form factor of a polymer crystalline-amorphous lamellar slab (Richter et al., 1996; Wang, 2004) and $S(Q)$ the structure factor of stacking slabs. An excess scattering which may originate from polymer blob structures (due to excluded volume interactions between chains in the amorphous brush-like region) or from polymers that are still in solution in a single-coil conformation is added. This is an important contribution to the scattering intensity at high $Q$ and is described by the Beaucage form factor [equation (2)] for $p=5 / 3$.

The lamellar slabs consist of crystalline cores of thickness $d$ surrounded on both sides by amorphous brushes of thickness $l_{\mathrm{b}}$ which are separated by a distance $D^{*}$ that, according to an above-mentioned assumption, fulfills the condition $D^{*}>d+$ $2 l_{\mathrm{b}}$. In a very simple approach, for both crystalline and amorphous layers a homogeneous rectangular density profile characterized by the corresponding contrast factors $\left(\Delta \rho_{\mathrm{c}}\right)^{2}$ and $\left(\Delta \rho_{\mathrm{b}}\right)^{2}$ is considered. The contrast factors account for the difference between the scattering length density of the solvent $\rho_{0}$ and that of the polymer $\rho_{\mathrm{p}}$ multiplied with the polymer volume fraction inside the crystalline $\left(\varphi_{\mathrm{c}}\right)$ and amorphous regions $\left(\varphi_{\mathrm{b}}\right)$, respectively. The form factor $P(Q)$ of the density profile along the direction perpendicular to the faces of the lamellar slab contains the contrast factors squared, which include the volume fractions of polymer $\varphi_{\mathrm{b}}$ and $\varphi_{\mathrm{c}}$ within the amorphous and crystalline layers, respectively $\left(\varphi_{\mathrm{c}}=1\right.$ is assumed).

Thus, the fitting procedure assumes a large number of free parameters $\left(d, l_{\mathrm{b}}, D^{*}, \sigma_{D^{*}}, \varphi_{\mathrm{s}}, \varphi_{\mathrm{b}}, R_{\mathrm{g}}^{\text {blob }}, G^{\text {blob }}\right)$ that must be determined in order to acquire a meaningful characterization of the complex morphology. In order to get reliable fits and values for the free structural and density parameters the modeling of the whole SANS pattern was done in a two-step procedure. In the initial step, which assumed only the fit of the high- $Q$ data, with the benefit of the data analysis in the Kratky approach (Fig. 8) the crystal thicknesses $d=110$ (15) and 93 (17) $\AA$ and the high- $Q$ excess scattering for the 9000 and 9007 OBCs were determined in a straightforward way. In the second step the crystalline-amorphous morphology was modeled using equation (4) with a reduced number of free 


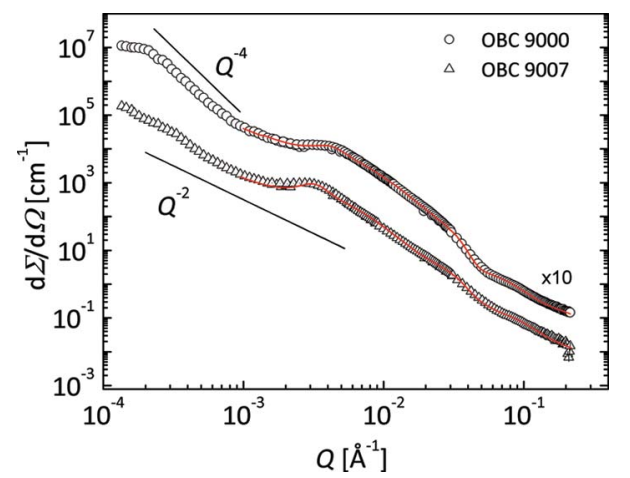

Figure 11

The experimental (symbols) and modeled (lines) small-angle neutron scattering cross sections from solutions of 9000 and 9007 OBCs in d-26 $(\varphi=1 \%)$ at $293 \mathrm{~K}$; for clarity the data from OBC 9000 are shifted vertically by a factor of ten.

parameters. A good fit of the whole scattering pattern for a reasonable set of values of the geometrical and density parameters is depicted in Fig. 11. The main parameters obtained in this case are $D^{*}=1750 \AA, \sigma_{D^{*}}=720 \AA, l_{\mathrm{b}}=180 \AA, \varphi_{\mathrm{b}}=0.12$ for the $9000 \mathrm{OBC}$ and $D^{*}=2150 \AA, \sigma_{D^{*}}=870 \AA, l_{\mathrm{b}}=220 \AA$, $\varphi_{\mathrm{b}}=0.30$ for the $9007 \mathrm{OBC}$.

The fitting approach represents a simplification of a more realistic case assuming a specific polymer density profile inside the amorphous region. This case, requiring a higher number of free parameters and thus a complicated and less reliable fitting procedure, was not considered here. Qualitatively, the results of the fitting are consistent with the chain characteristics. The 9007 OBC, the polymer with the lower crystallinity and thus large soft block content, presents a larger thickness of the amorphous layer and a higher polymer volume fraction in the amorphous region than the more crystalline 9000 OBC. The latter yields thicker crystalline lamellae and more compact macro-aggregates. Also, it is worth noting that the needle-like wax structures show a thickness of about $0.5 \mu \mathrm{m}$ (Figs. $5 d$ and $5 e$ ), which is in good agreement with the total thickness of the core-brush morphology of $9000 \mathrm{OBC}$, and this morphology presumably templates the final wax structure.

The main morphological characteristics obtained by SANS, namely the unusually large interlamellar distance and the peculiarity that the crystalline-amorphous lamellae are well separated, are similar to those observed for the morphology of OBCs crystallized from the melt. There, much larger interlamellar spacings than expected for traditional block copolymers of similar $M_{\mathrm{W}}$ were reported (Hustad et al., 2009; Jin et $a l ., 2010)$. In these cases, the large interlamellar spacing was considered to be a direct consequence of the polydisperse character of the OBCs, which causes anomalously thick amorphous regions. It was observed that in melts, following perturbations occurring during the sample preparation, a fraction of the molecules with a large mismatch in block length are driven away from the lamellar interface and swell the domain preferred by the longer blocks (Hustad et al., 2009). It was also observed that more amorphous phases are rejected outside of the lamellar stacks (Tong et al., 2012). Thus, it was demonstrated (Jin et al., 2010) that smaller perturbations favor the expelling of unbalanced molecules for which the entropy lost by the longer block opposes the enthalpy gained by surrounding the short blocks with similar segments. In the case of the morphologies formed by the OBCs in solution a similar behavior might also be responsible for the observed large separation of the lamellar slabs. The high polydispersity degree (both in block length and in the number of blocks per chain) and relatively low crystallinity would favor the formation of broad amorphous phases and narrow crystalline layers. The increase of the interlamellar spacing in time or with lowering temperature would be a consequence of the gradual swelling of the amorphous phase based on the continuous expelling of unbalanced molecules, which ultimately will return in the solution.

Finally, as already demonstrated by the incipient optical microscopy observations of mixed polymer-wax aggregates, the crystalline-amorphous $\mathrm{OBC}$ assemblies may represent an appropriate environment for dictating the habit and controlling the size of the waxy crystals grown in hydrocarbon solutions. The usefulness of the OBCs in such applications will be the subject of a future contrast-matching SANS structural study on ternary polymer-wax-solvent systems.

\section{Conclusions}

The chain properties and the self-assembly behavior of two olefin block copolymers obtained by chain-shuttling chemistry were investigated by combined wide- $Q$-range small-angle neutron scattering, optical microscopy with crossed polarizers and DSC. The complex morphologies yielded with decreasing temperature multiple structural levels that are hierarchically organized and with a size range from $10 \AA$ to tens of micrometres. The peculiarities observed in the structure and morphology of the OBC macro-aggregates are a consequence of the chain characteristics and of the polydispersity in block length and in the number of blocks per chain. These selfassembling multi-block polymers show the formation of soluble complex hydrocarbon architectures that are converted to single coils above $\sim 378 \mathrm{~K}$. This reversible behavior suggests that the INFUSE block copolymers can have applications as downstream petroleum additives. These could include uses such as wax crystal and viscosity index modifiers.

\section{Acknowledgements}

The optical microscopy and DSC investigations were performed at the Materials Science Laboratory of Heinz Maier-Leibnitz Centre (MLZ), Technische Universität München (TUM), in collaboration with Sebastian Busch (TUM) and Armin Kriele (Helmholtz-Zentrum Geestacht,

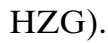

\section{References}

Akpalu, Y. V. (2010). Polym. Rev. 50, 1-13.

Arriola, D. J., Carnahan, E. M., Hustad, P. D., Kuhlman, R. L. \& Wenzel, T. T. (2006). Science, 312, 714-719.

Beaucage, G. (1996). J. Appl. Cryst. 29, 134-146. 
Dias, P., Lin, Y. J., Poon, B., Chen, H. Y., Hiltner, A. \& Baer, E. (2008). Polymer, 49, 2937-2946.

Gläser, W. \& Petry, W. (2000). Physica B, 276-278, 30-32.

Goerigk, G. \& Varga, Z. (2011). J. Appl. Cryst. 44, 337-342.

Gránásy, L., Pusztai, T., Tegze, G., Warren, J. A. \& Douglas, J. F. (2005). Phys. Rev. E, 72, 011605.

Green, P. F. (2001). Adv. Colloid. Int. Sci. 94, 53-81.

Hammouda, B. (2010). J. Appl. Cryst. 43, 1474-1478.

Hustad, P. D., Marchand, G. R., Garcia-Meitin, E. I., Roberts, P. L. \& Weinhold, J. D. (2009). Macromolecules, 42, 3788-3794.

Jin, J., Du, J., Xia, Q., Liang, Y. \& Han, C. C. (2010). Macromolecules, 43, 10554-10559.

Kamdar, A. R., Wang, H. P., Khariwala, D. U., Taha, A., Hiltner, A. \& Baer, E. (2009). J. Polym. Sci. B Polym. Phys. 47, 1554-1572.

Magil, J. H. (2001). J. Mater. Sci. 36, 3143-3164.

Radulescu, A., Fetters, L. J. \& Richter, D. (2008). Adv. Polym. Sci. 210, 1-101.

Radulescu, A., Fetters, L. J. \& Richter, D. (2012). Crude Oil Emulsions - Composition Stability and Characterization, edited by M. S. Abdel-Raouf, pp. 205-230. Rijeka: Intech.

Radulescu, A., Pipich, V., Frielinghaus, H. \& Appavou, M. S. (2012). J. Phys. Conf. Ser. 351, 012026.

Radulescu, A., Pipich, V. \& Ioffe, A. (2012). Nucl. Instrum. Methods Phys. Res. Sect. A, 689, 1-6.
Radulescu, A., Schwahn, D., Monkenbusch, M., Fetters, L. J. \& Richter, D. (2004). J. Polym. Sci. B Polym. Phys. 42, 31133132.

Radulescu, A., Schwahn, D., Stellbrink, J., Kentzinger, E., Heiderich, M., Richter, D. \& Fetters, L. J. (2006). Macromolecules, 35, 37623768.

Radulescu, A., Schwahn, D., Stellbrink, J., Monkenbusch, M., Fetters, L. J. \& Richter, D. (2011). J. Polym. Sci. B Polym. Phys. 49, 144158.

Richter, D., Schneiders, D., Monkenbusch, M., Willner, L., Fetters, L. J., Huang, J. H., Lin, M., Mortensen, K. \& Farago, B. (1996). Macromolecules, 30, 1053-1068.

Schmidt-Rohr, K. (2007). J. Appl. Cryst. 40, 16-25.

Schwahn, D., Richter, D., Wright, P. J., Symon, C., Fetters, L. J. \& Lin, M. (2002). Macromolecules, 35, 861-870.

Shan, C. L. P. \& Hazlitt, L. G. (2007). Macromol. Symp. 257, 8093.

Tong, Z. Z., Xu, J. T., Xia, S. J. \& Fan, Z. Q. (2012). Polym. Int. 62, 228-237.

Wang, H. P. (2004). J. Polym. Sci. B Polym. Phys. 42, 3133-3147.

Wang, H. P., Chum, S. P., Hiltner, A. \& Baer, E. (2009). J. Appl. Polym. Sci. 113, 3236-3244.

Wang, H. P., Khariwala, D. U., Cheung, W., Chum, S. P., Hiltner, A. \& Baer, E. (2007). Macromolecules, 40, 2852-2862. 